\title{
Human factors methods to reduce medication error: using task analysis in a pediatric and adult pharmacy
}

\author{
Nicole E. Werner, ${ }^{*}$ Erik T. Nelson, ${ }^{a}$ and Deborah A. Boehm-Davis ${ }^{\mathrm{a}}$ \\ ${ }^{a}$ Psychology Department, George Mason University, 4400 University Drive, MS3F5, Fairfax, VA 22030, United \\ States.
}

\begin{abstract}
Medication error is an issue that no hospital is immune from, leading to 7,000 deaths and 1.3 million patient injuries each year. The purpose of this study was to decrease the risk and occurrence of medication errors by analyzing the hospital pharmacy. Task analyses were performed and it was found that communication, expectation, and procedural issues were leading to the occurrence of the most common type of medication error in the pharmacy. Recommendations were made to improve the process and reduce the occurrence of this type of error.
\end{abstract}

Keywords: Patient safety, medication error, task analysis, pharmacy

\section{Introduction}

According to the 1999 Institute of Medicine report, "To err is human," human error in medicine leads to the deaths of up to 98,000 people per year [3]. Medication errors are defined as an error that occurs during any part of the medication dispensing process. This process starts with the provider who writes the prescription, goes through the pharmacy who transcribes and dispenses the prescription, and ends with the nurses who administer the prescription.

Medication error is an issue that no hospital system is immune from. In fact, medication errors have been reported to contribute to 7,000 deaths and 1.3 million patient injuries each year [1]. In addition to harm to patients, medication error also leads to an enormous expense for the healthcare system. In 2000, $\$ 177.4$ billion was spent for injuries caused by medication errors [2]. Because of the high cost to patient's well being, as well as monetary cost to the healthcare system, it is important to develop and apply new ways of reducing medication errors.
This study set out to identify potential causes of medication errors for patients served by an adult and pediatric satellite pharmacy within a large suburban hospital system. Human factors and task analysis techniques were used to identify these errors.

\section{Practice Innovation}

Observations of pharmacy staff initially began with 30 minute to two hour sessions followed by unstructured interviews regarding processes and culture. Later, unstructured interviews were conducted with the nursing staff on the several floors/wings of the hospital with regard to their perceptions of the medication preparation process, as well as the transfer of medications from the pharmacy to the nursing floor. From these information gathering techniques, two task analyses were conducted; a Barrier Analysis and an Operational Sequence Diagram.

*Nicole E. Werner. E-mail: nwerner2@masonlive.gmu.edu 


\section{Task Analyses Findings}

A barrier analysis was conducted to identify what barriers are currently in place to prevent medication errors. The barrier analysis revealed some interesting initial findings. First, when reviewing the error reporting database for the hospital, missing dose errors were identified as the most common type of error. A missing dose error is reported if the medication is not available to the nurse at the time of administration. However, when analyzing the barriers in place to combat medication error, it became clear that the current barriers (e.g., double-check requirements, interruption awareness signs) focused on wrong patient or wrong drug type errors which occurred less frequently. These errors are of higher risk to the patient, and should have safety barriers in place against administration error. However, these are not the only medication-error types that affect patient safety. Although missing dose errors typically lead to less severe consequences than errors such as giving the wrong medication to a patient, this is not always the case. A missing dose error can lead to a long delay in a patient receiving a much-needed medication, the missing of the dose altogether, and even double dosing the patient with the medication. Both symbolic and immaterial barriers were in place to protect the pharmacy from those errors typically labeled as low frequency/high consequence (e.g., giving a medication to the wrong patient). However, these barriers had little to no effect on the most frequent type of medication error associated with this pharmacy - the missing dose error.

Because the missing dose error has the highest frequency, it became the focus of our analyses going forward. When examining the process of medication administration, the transfer of medications from the pharmacy to the nurses and patients is an essential piece. For this reason, we chose to construct an Operation Sequence Diagram (OSD) of the medication transfer process. An OSD is designed to identify how individual operators work together to complete a complex task, and can detect possible inefficiencies within the system.

From this analysis, we discovered that there are actually three systems involved in delivering medication to patients. There is the satellite pharmacy and nursing units as previously described, as well as an over-night pharmacy (operating 2300 until 0600). The OSD revealed a disconnect between these three systems. Nurses in their respective units expect to administer the first round of medication for the day at 0800. However, procedure dictates that the over- night pharmacy begin transferring prescriptions to the satellite pharmacy at 0400 , such that when the staff arrive to the satellite pharmacy at 0600 , they have a back $\log$ of prescriptions to fill, in addition to the daily prescriptions. Due to this high workload, the satellite pharmacy does not plan to deliver any medications to the nursing units until 0900. Because nurses expected the medications for their patients by 0800 and the pharmacy did not generally deliver until 0900, nurses frequently recorded the first dose of medication to be a missing dose and they proceeded to call the pharmacy for a STAT order, thus causing more of a back $\log$ in prescriptions to be filled. Finally, at the end of the day, pharmacists were finding that many of the STAT orders that they had filled were returned to them because the same prescriptions were being filled more than once. Interviews with pharmacists, nurses, and those that deliver the medications to the floors from the pharmacy revealed that these STAT orders were being returned because the nurse would typically call them in during that back$\log$ time in the morning. Meanwhile, the original order would show up and was immediately given to the patient. Then, after the original medication was administered, the STAT order would show up, only to be returned later.

As already mentioned, administering a medication late is technically an error, yet it tends to be considered less serious than other errors such as wrong $\mathrm{pa}$ tient or wrong drug. However, nurses calling in STAT orders while the original medication is on its way can lead to more serious consequences. Most notably, there is a potential for inadvertently administering a patient a double dose. After analyzing several of the nurse stations, it became clear that there was not a hospital wide procedure for dealing with medications that need to be returned to the pharmacy. Medications that needed to be returned were found on countertops, in bins, and even in medication dispensing machines. If the pharmacist delivers a STAT dose to a nurse, the original prescription can still be filled if it is in that back-log. If that prescription is delivered, it will sit in the patient's bin until the next pharmacy delivery comes and someone notices that that it should not be there. However, if a new shift starts, and a new nurse sees medication that has yet to be delivered to the patient sitting in the medication bin, she might think that the dose was missed, and administer it to the patient (causing a double dose). 


\section{Recommendations}

Based on the task analyses, changes in procedure can be implemented in order to reduce the risk of medication error, and most likely reduce the occurrence of medication error in this pharmacy. These changes will necessitate a focus on the missing dose error which has previously been overlooked, most likely due to the lower instance of sentinel events (events leading to a patient death) associated with it. However, while the potential risks associated with this type of error may be viewed as lower than that of others (e.g., wrong patient or wrong medication errors), there are still many risks associated with this type of error such as: patients in pain, missed meals, patient dissatisfaction, and the patient receiving a double dose of medication.

The first recommendation was to try and match the expectations of the pharmacy with those of the nursing staff. This could be accomplished by changing the time that the pharmacy needs to distribute medications, changing the timing for prescribing medications, or having the overnight pharmacy be responsible for prescriptions for a longer period of time to lessen the back-log of prescriptions when the satellite pharmacists arrive in the morning.

The second recommendation was to develop procedures to be followed when a nurse wants to request a missing medication. Rather than just ordering a new medication when a missing dose is noticed, nurses should notify the pharmacy without causing excessive disruption, and the pharmacy can let them know how and when the medication will be delivered. One method could use a computer system so that the nurse can track the progress of the medication as it goes through the pharmacy and delivery process.

The third recommendation was to develop well defined and hospital wide procedures to follow that stipulate how to handle leftover or unused medications. The medication bins and medication area should be checked regularly to make sure there are no extra medications - especially before a shift change. If medications are not used, the pharmacy should retrieve them before the nurse's next med pass. Alternatively, there could be a clearly marked container for unused medications in the medication area that the nurses can fill for the pharmacy to pick up at a later time.

\section{Next Steps}

Using a Human Factors approach to analyzing the medication error issue led to some interesting initial findings, and pointed to areas in which improvement is necessary. The next steps of this process are to implement the changes suggested by the task analyses and monitor the success of the changes via the error reporting database. Once the efficacy of the changes has been verified, the goal is to apply these task analysis techniques to other pharmacies within the hospital system in order to identify other potential changes that can be implemented.

Applying Human Factors techniques such as task analysis can be integral to improving patient safety as well as worker safety. Although these techniques are invaluable to the improvement of current work systems, they should also be considered when designing procedures in the pharmacy and other areas of medication administration. This study has demonstrated that through the use of Human Factors techniques, efficiency in medication administration can be improved, and the source of potential errors can be identified to help reduce future error.

\section{References}

[1] Committee on Quality of Health Care in America. (2000). Error in health care: A leading cause of injury and death, 26-48.

[2] Ernst, F. R. \& Grizzle, A. J. (2001). Drug related morbidity and mortality: Updating the cost for illness model. Journal of the American Pharmacy Association, 41, 192-199.

[3] Institute of Medicine. (1999). To Err is Human: Building a safe health care system. Washington DC: National Academies Press. 